\title{
CORRECTION
}

\section{Correction to: Gender Differences and Prevalence of Mental Health Problems in Students of Healthcare Units}

\author{
Mehnaz Nuruddin Gitay ${ }^{1}$. Sarah Fatima ${ }^{1}$. Shahama Arshad ${ }^{1}$. Basmah Arshad ${ }^{1}$. Ahsan Ehtesham ${ }^{1}$. \\ Muhammad Ameen Baig ${ }^{1}$. Muhammad Furqan Ilyas ${ }^{1}$. Shaad Farhat Rizvi ${ }^{1}$. Qadeer Farooqui ${ }^{1}$. \\ Muhammad Masroor ${ }^{2} \cdot$ Zeba Haque $^{1}$
}

Published online: 20 September 2018

๑) Springer Science+Business Media, LLC, part of Springer Nature 2018

\section{Correction to: Community Mental Health Journal} https://doi.org/10.1007/s10597-018-0304-2

The original version of this article unfortunately contained a typo in the author name. The co-author name should be Ahsan Ehtesham instead it was published as Ahsan Ehtisham.

The original article has been corrected.

The original article can be found online at https://doi.org/10.1007/ s10597-018-0304-2.

Mehnaz Nuruddin Gitay

quick_gitay@hotmail.com

Sarah Fatima

Sarahfatima07@hotmail.com

Shahama Arshad

Shahama_62@hotmail.com

Basmah Arshad

arshad.60@gmail.com

Ahsan Ehtesham

tariq_ch@hotmail.com

Muhammad Ameen Baig

ameen_baig_91@hotmail.com

Muhammad Furqan Ilyas

furqanmemon@hotmail.com
Shaad Farhat Rizvi srizvi32@gmail.com

Qadeer Farooqui hfaruki@hotmail.com

Zeba Haque

z.haque@duhs.edu.pk

1 Department of Biochemistry, Dow International Medical College (Ojha Campus), Dow University of Health Sciences, Karachi, Pakistan

2 Department of Medicine, Dow International Medical College, Dow University of Health Sciences, Karachi, Pakistan 